\title{
Politique
}

Politique

\section{L'étude des partis au Québec depuis 1970}

\section{Vincent Lemieux}

Numéro 23, hiver 1993

Tendances de la science politique au Québec

URI : https://id.erudit.org/iderudit/040747ar

DOI : https://doi.org/10.7202/040747ar

Aller au sommaire du numéro

Éditeur(s)

Société québécoise de science politique

ISSN

0711-608X (imprimé)

1918-6584 (numérique)

Découvrir la revue

Citer cet article

Lemieux, V. (1993). L'étude des partis au Québec depuis 1970. Politique, (23), 43-57. https://doi.org/10.7202/040747ar

\section{Résumé de l'article}

Les travaux publiés, depuis 1970, sur les partis politiques au Québec ont été nombreux et divers, mais leur caractère cumulatif demeure limité en ce sens que peu d'auteurs ont repris la démarche ou les conclusions d'autres auteurs. Un petit nombre de jeunes politologues ont fait de l'étude des partis leur champ principal de recherche, et les préoccupations théoriques sont demeurées relativement rares. La situation est à peu près la même aux États-Unis et ailleurs dans le monde.
Ce document est protégé par la loi sur le droit d'auteur. L'utilisation des services d’Érudit (y compris la reproduction) est assujettie à sa politique d'utilisation que vous pouvez consulter en ligne.

https://apropos.erudit.org/fr/usagers/politique-dutilisation/ 


\title{
L'ÉTUDE DES PARTIS AU QUÉBEC DEPUIS 1970
}

\author{
Vincent Lemieux \\ Université Laval
}

Les travaux publiés, depuis 1970, sur les partis politiques au Québec ont été nombreux et divers, mais leur caractère cumulatif demeure limité en ce sens que peu d'auteurs ont repris la démarche ou les conclusions d'autres auteurs. Un petit nombre de jeunes politologues ont fait de l'étude des partis leur champ principal de recherche, et les préoccupations théoriques sont demeurées relativement rares. La situation est à peu près la même aux ÉtatsUnis et ailleurs dans le monde.

Le bilan des études sur les partis politiques au Québec depuis 1970, présenté ici, ne comprend pas le vaste domaine des études électorales. Trois questions sont sous-jacentes à ce bilan. D'abord, les connaissances acquises présentent-elles un caractère cumulatif? Ensuite, l'intérêt des chercheurs pour les partis a-t-il augmenté ou diminué? Enfin, qu'en est-il de l'apport théorique de ces études? Ces questions seront soulevées au cours de l'article et abordées de nouveau en conclusion.

Ces interrogations n'intéressent pas seulement le Québec. Depuis 1970, à travers le monde, l'étude des partis n'est plus un des champs dominants de la science politique. Les politiques publiques et les groupes de pression sont, parmi d'autres, deux domaines actuellement plus développés. En fait, il n'y a pas eu depuis longtemps de contributions importantes à la théorie des partis politiques, sauf peut-être en ce qui concerne les coalitions gouvernementales. De même, si les études électorales continuent d'être nombreuses, la recherche sur l'organisation des partis, elle, ne s'est pas accrue depuis les travaux classiques d'Ostrogorski, de Michels 
et de Duverger. Peu de candidats au doctorat et de jeunes chercheurs choisissent les partis comme objet principal de leurs recherches. Cette désaffection envers ce domaine chez les politologues correspond sans doute à celle que l'on remarque dans les sociétés.

Les ouvrages généraux sur les partis et les systèmes de partis au Québec

Commençons par les ouvrages généraux consacrés aux partis politiques du Québec. On peut les classer en trois catégories. Dans la première, prend place l'ouvrage de Réjean Pelletier (1989) sur les partis provinciaux du Québec de 1944 a 1970. II repose surtout sur des recherches originales portant sur les programmes et le personnel politique, mais il aborde également, de façon plus générale, d'autres aspects de l'action des partis. La deuxième catégorie comprend des études générales sur le système des partis. Citons les travaux de Robert Boily $(1976,1982)$ sur l'évolution du système des partis provinciaux où l'auteur établit l'arbre généalogique des partis qu'il reprend dans une publication ultérieure (Bernier et Boily, 1986, p. 244-245). On retrouve également dans cette catégorie des présentations générales du système des partis provinciaux au Québec (Lemieux 1971, 1976, 1992); elles s'attachent aux composantes et aux fonctions des partis. Enfin, une troisième catégorie inclut deux ouvrages collectifs, I'un dirigé par Pelletier (1976) et l'autre par Lemieux (1982), qui touchent à différents objets d'étude sur lesquels nous reviendrons. Tous ces travaux ont des visées descriptives ou analytiques, plutôt que théoriques. Elles utilisent à cette fin un certain nombre de concepts, variables selon les auteurs.

Ces trois catégories d'ouvrages portent exclusivement sur le système des partis provinciaux. Le système des partis fédéraux du Québec n'a pas fait l'objet d'études comparables. Les travaux plus spécialisés montrent la même inégalité. Les partis provinciaux ont aussi reçu plus d'attention que les partis fédéraux. Et parmi les partis provinciaux, c'est l'Union nationale qui vient en tête, en bonne partie à cause de la fascination exercée par son chef fondateur, Maurice Duplessis. En plus des biographies de Rumilly (1973) et de Black 
(1977), signalons trois ouvrages : celui de Quinn (1978), qui insiste sur le nationalisme du parti, celui de Boismenu (1981), qui traite surtout de la politique économique de l'Union nationale sous Duplessis, et celui de Cardinal, Sauvageau et Lemieux (1978), basé sur une série d'émissions radiophoniques portant sur l'Union nationale et composées de témoignages de politiciens et d'observateurs politiques. Les ouvrages de Quinn et de Boismenu ont un caractère plus théorique que les autres en ce qu'ils cherchent à démontrer des thèses formulées de façon explicite.

Fait étonnant, le Parti libéral, qui est pourtant le seul parti à avoir subsisté depuis le $\mathrm{XIX}^{\bullet}$ siècle, a jusqu'à maintenant fait l'objet d'études plus partielles que l'Union nationale. Dupont (1973) a publié un livre fort utile sur les relations entre l'Église et l'État sous Taschereau dont Bernard Vigod a écrit une biographie (1986) qui éclaire le Parti libéral du premier tiers du siècle. Dale Thomson a fait de même pour Jean Lesage (1984). De plus, les deux premiers colloques sur les leaders politiques du Québec contemporain, tenus par I'UOAM, ont été suivis de publications sur Lapalme (Léonard, 1988) et sur Lesage (Comeau, 1989) où des témoins et des universitaires apportent sur le Parti libéral des années 1950 et 1960 , plusieurs informations. Notre propre ouvrage sur le Parti libéral du Québec (Lemieux, 1993), porte principalement sur les alliances, les rivalités et les neutralités qu'entretient le parti avec son entourage externe et interne. Un appendice théorique propose une mise en forme de ces liens d'alliance, de rivalité et de neutralité.

Même s'il existe depuis moins longtemps que le Parti libéral, le Parti québécois a suscité beaucoup d'intérêt. Déjà dans les années 1970, Vera Murray lui consacrait un ouvrage (1976) portant sur le programme et les structures du parti ainsi que sur le leadership de René Lévesque. André Larocque avait publié plus tôt (1971) un petit livre «engagé" traitant principalement de l'organisation, de l'action et des structures du parti. Graham Fraser (1984) s'intéresse surtout aux principaux dirigeants du parti et aux décisions qu'ils ont été amenés à prendre. D'esprit journalistique, son livre repose surtout sur l'observation directe et sur des entrevues. Tous 
ces ouvrages sur le Parti québécois ont peu ou pas du tout de visées théoriques.

En ce qui a trait aux tiers partis, signalons l'imposant ouvrage de Paul-André Comeau consacré au Bloc populaire (1982) et basé en bonne partie sur des entrevues menées auprès d'anciens membres de ce parti. Dans la série sur les leaders politiques, le tome consacré à André Laurendeau (Comeau et Beaudry, 1990) contient également de l'information sur le Bloc populaire. Quant aux militants du RIN, ils ont fait l'objet d'une étude réalisée par Réjean Pelletier (1974), à partir de données recueillies par questionnaire. Gérald Bernier (1982) a, pour sa part, publié une brève étude du Parti patriote, tandis que Robert Comeau et Bernard Dionne (1989) ont écrit un ouvrage sur les communistes au Québec.

Les partis fédéraux au Québec, tout comme l'a été le système de ces partis, ont été négligés, du moins par les politologues (on trouve cependant quelques aperçus dans Gagnon et Tanguay, 1989). Les historiens s'y sont cependant intéressés. Les ouvrages de Bélanger sur Lamarche (1984) et Laurier (1986) ont éclairé la vie des partis fédéraux à la fin du $X I X^{\circ}$ siècle et au début du $X X^{\circ}$. Celui de Jones (1980), qui traite de l'hégémonie libérale au Québec du temps de Laurier et de King, insiste sur les grands débats qui ont agité les partis fédéraux. Signalons aussi l'analyse de La Terreur (1973) sur les tribulations des conservateurs au Québec. À côté de ces contributions, celles des politologues sont plutôt minces. Trois ouvrages seulement se démarquent : celui de Stein (1973) sur le Crédit social au Québec, celui de Lamoureux (1985) sur le NPD et le Québec de 1958 à 1985, et l'ouvrage dirigé par Yves Bélanger et Dorval Brunelle (1988). Consacré à l'ère des libéraux, ce dernier porte sur l'exercice du pouvoir dans différents secteurs par leur gouvernement, de 1963 à 1984. On doit ajouter à cette liste I'ouvrage du sociologue Maurice Pinard (1975) sur la montée du Parti du Crédit social au Canada, l'un des plus importants de notre bilan pour sa contribution théorique à l'étude des tiers partis.

Les partis municipaux de Montréal et de Québec ont fait I'objet de quelques travaux, dont ceux de Louise Quesnel (1976, 1982) et de quelques-uns des collaborateurs de 
l'ouvrage publié sous la direction de Guy Bourassa et de Jacques Léveillée (1986) et qui analyse le système politique de Montréal. Plus récemment, Louise Quesnel et Serge Belley (1991) ont fait paraître un livre traitant des élections et des partis municipaux à Québec, et Serge Belley (1992) a publié une étude comparative des partis et des élections à Montréal et à Québec. Ces travaux centrés sur les partis municipaux ne manifestent pas de préoccupations théoriques.

\section{Les ouvrages thématiques}

On dispose d'autres travaux consacrés à certains aspects de l'action des partis, mais plusieurs ouvrages déjà signalés traitent aussi de l'un ou l'autre de ces thèmes. Les politiques publiques, par exemple, occupent une grande place chez Boismenu (1981), Dupont (1973), Jones (1980), Bélanger et Brunelle (1988). Ce sont sans doute les programmes et, plus généralement, le discours politique qui ont le plus retenu l'attention des chercheurs et qui ont donné lieu à l'application des méthodes les plus variées. Réjean Pelletier a fondé sur l'analyse des programmes une bonne partie de ses synthèses concernant les partis $(1980,1989)$ : quelques grandes oppositions (interventionnisme/non-interventionnisme, collectivisme/individualisme) inspirent ses analyses. Les programmes électoraux ont fait l'objet d'analyses de contenu effectuées par Mellos (1970), Latouche (1976), Lafleur (1985) et Monière (1988, 1991). Celui-ci, dans son ouvrage de 1988, essaie de vérifier la fiabilité du discours électoral en étudiant la concordance entre les promesses faites lors de la campagne électorale et les réalisations du gouvernement conservateur de 1984 à 1988 . Hamel et Thériault (1975) ont étudié la fonction tribunitienne dans le discours créditiste à l'Assemblée nationale, de 1970 à 1973, tandis que Manon Tremblay $(1989,1992)$ s'est intéressée au contenu féministe du discours des élues du $31^{\circ}$ Parlement du Québec. Bourque et Duchastel (1988) ont analysé pour leur part les thèmes contradictoires de la tradition et du progrès dans le discours politique sous le régime de Duplessis. Landry, quant à lui, a conçu une méthode inspirée de la théorie des choix collectifs, méthode qui vise à établir les différentes catégories d'avanta- 
ges et de coûts proposés aux divers destinataires dans les programmes électoraux des partis (Landry et Duchesneau, 1987).

Landry (1990) a appliqué la même méthode dans son analyse du sommaire des lois qu' ont fait adopter les différents gouvernements à Québec depuis 1960. Nous avons aussi analysé les lois adoptées depuis 1945 par les gouvernements de partis, afin d'établir les relations de pouvoir entre destinateurs et destinataires (Lemieux, 1991). Ces deux analyses s'inspirent respectivement des modeles theoriques des choix rationnels et du contrôle.

Les travaux ayant trait au personnel politique des partis se poursuivent avec la participation des deux principaux animateurs, Robert Boily (1969) (voir aussi Bernier et Boily, 1986) et Réjean Pelletier (1984, 1989, aussi Pelletier et Crête, 1988, Pelletier et Tremblay, 1992). Leurs étudiants et assistants ont publié quelques articles et travaux, mais on ne peut pas dire que ce domaine est en expansion à l'heure actuelle. Rappelons aussi l'ouvrage de Gélinas (1969) sur les parlementaires et l'administration au Québec, très riche d'informations sur la fin des années 1960. Louis Massicotte (1989) pour sa part, dans son analyse de la cohésion et de la dissidence dans les partis à l'Assemblée législative du Québec depuis 1867, a montré que la dissidence était plus fréquente au $\mathrm{XIX}$ ' siècle qu'elle ne I'a été depuis.

Les élus et les candidats défaits ne sont pas les seuls acteurs partisans dont les caractéristiques ou les comportements intéressent les chercheurs. Renaud (1973) a étudié les motivations des militants de I'Union nationale dans une circonscription. Nous avons déjà signalé l'ouvrage que Réjean Pelletier (1974) a consacré aux militants du RIN. Legault, Desrosiers et Tardy (1988) ainsi que Maillé et Tardy (1988) ont analysé les différences entre les hommes et les femmes dans les partis provinciaux et dans les partis municipaux. Jean-Pierre Beaud (1982) s'est intéressé à différentes catégories de personnels partisans du Parti québécois : adhérents, délégués au congrès national, membres de l'exécutif national, députés, ce qui lui a permis d'établir certains traits de la "pyramide" péquiste. Pierre O'Neill et Jacques Benjamin (1978) élargissent le cercle et montrent 
I'influence respective des conseillers partisans, des conseillers gouvernementaux (ministres et hauts fonctionnaires) et des fabricants d'image auprès des premiers ministres Lesage, Johnson, Bertrand, Bourassa première manière, et Lévesque.

Benjamin avait écrit auparavant un livre (1975) sur la "fabrication" d'un premier ministre québécois par les spécialistes du *marketing". Dans ce courant se situent les ouvrages de Robert Bernier (1988 et 1991 ) sur le *marketing" gouvernemental au Québec de 1929 à 1985 et sur le "marketing" électoral des partis fédéraux, lors des élections générales de 1988.

Le patronage politique des partis provinciaux québécois, de 1944 à 1972, est le sujet d'une étude de Lemieux et Hudon (1975), fondée sur l'analyse de la presse écrite et sur des entrevues. Le cas du Québec est aussi traité dans notre étude comparative du patronage politique (1977).

Le financement des partis n'est pas sans relation avec les pratiques de patronage. $A$ côté des travaux d'Angell (1982, 1987), signalons l'étude très fouillée de Louis Massicotte (1991) sur le financement des partis provinciaux du Québec depuis l'adoption de la loi de 1977.

Enfin, l'organisation interne des partis a fait l'objet de peu de travaux depuis vingt ans. Les ouvrages collectifs de Pelletier (1976) et de Lemieux (1982) en contiennent quelques-uns, mais presque tous sont basés sur des données recueillies avant 1970 . On trouve quelques aperçus sur ce thème dans des travaux déjà cités, en particulier dans ceux de la série de I'UOAM sur Lapalme et Lesage. Mais tout compte fait ce domaine de recherche est très négligé. Dans un article (Lemieux, 1988), nous avons proposé une façon nouvelle, inspirée d'une théorie du contrôle, d'étudier les statuts des partis, en prenant pour exemple ceux du NPD. Réjean Pelletier (1991) pour sa part a fait une étude générale des structures et du fonctionnement des partis politiques canadiens.

Finalement, on compte peu de contributions théoriques significatives dans ces quelques travaux sur l'organisation des partis, et, plus généralement, dans les ouvrages thématiques. Cette situation est d'ailleurs à l'image de la science des partis, telle qu'elle a évolué depuis 1970. 


\section{Bilan et perspectives}

Malgré tout, les publications consacrées aux partis ont été diverses et relativement nombreuses depuis 1970. Leur production a été constante au cours de la même période. Par contre leur caractère cumulatif a été très limité, en ce sens que peu d'auteurs ont repris la démarche ou les conclusions d'autres auteurs pour faire avancer les connaissances dans un domaine donné. C'est peut-être seulement dans les études sur le personnel politique qu'il y a eu un certain cumul, sans doute parce que ce domaine s'y prête mieux. Même dans un secteur aussi largement exploré que celui des programmes et du discours politique, les travaux de recherche ne présentent à peu près pas de caractère cumulatif.

Comme nous l'avions présumé au départ, peu de jeunes chercheurs dans nos départements de science politique ou ailleurs font de l'étude des partis politiques leur champ principal ou un de leurs champs secondaires de recherche. II ne faut sans doute pas s'en étonner si l'on considère que, dans la science politique internationale, l'étude des partis politiques est un domaine qui se développe et se renouvelle très peu. Les jeunes chercheurs qui s'intéressent au Québec sont attirés, avec raison, par des courants plus dynamiques ou plus centraux de la science politique contemporaine, ce qui a des conséquences sur le plan méthodologique. En effet, $c^{\prime}$ est généralement lorsqu'on est jeune et plus disponible que l'on observe de près le fonctionnement des partis, en assistant à des congrès, à des assemblées d'investiture, à des assemblées électorales. C'est à ce moment-là que l'on réalise des entrevues avec des partisans ou que l'on expérimente des méthodes nouvelles pour analyser le matériel recueilli. Cette activité a décliné au cours des deux dernières décennies, et même s'il y a quelques signes encourageants à l'horizon, on ne peut pas dire que l'heure des nouveaux départs et du grand renouvellement est proche.

Les préoccupations théoriques ont toujours été et sont encore relativement rares, d'où l'indigence théorique dont souffre la science politique des partis. Les ouvrages généraux, inspirés d'une ou de quelques théories, sont rares (Bernard, 1976, Pinard, 1976, Lemieux, 1985). Dans les domai- 
nes plus particuliers, peu de chercheurs (Boismenu, 1981, Landry et Duchesneau, 1987, Landry, 1990, Pinard, 1975) ont eu recours pour leurs analyses à des théories existantes ou ont cherché à en créer de nouvelles (Stein, 1973, Lemieux, 1993). Les systèmes de partis ne sont pas des systèmes comme les autres, et les partis sont des organisations différentes des autres, ce qui rend difficiles des avancées qui s'inspireraient de théories ayant été appliquées à d'autres systèmes ou organisations. C'est toutefois le défi qu'il faudra bien relever si l'on veut faire de l'étude des partis et de leurs systèmes un courant qui attire plus de jeunes chercheurs ambitieux que ce n'est le cas actuellement au Québec et à travers le monde.

S'il fallait indiquer, pour le Québec, quelques champs de recherche dont l'exploration ferait avancer la science des partis, on pourrait en citer trois. En tout premier lieu, il y aurait l'organisation des partis, domaine qui permettrait de renouveler ou de développer les méthodes de recherche dont nous avons parlé précédemment. Ce n'est pas parce que l'étude de l'organisation des partis est exigeante sur ce plan qu'il faut pour autant la négliger. Un deuxième secteur de recherche à développer est celui de la contribution des partis aux politiques publiques. Contribution au moyen des programmes électoraux que les partis proposent, des lois qu'ils font adopter, et du pouvoir plus ou moins grand qu'ils exercent dans les processus d'émergence, de formulation et de mise en œuvre des politiques. Ce domaine a donné lieu à un grand nombre de recherches et de débats depuis une vingtaine d'années, notamment en Europe. Enfin, troisième domaine, relié au précédent : le déclin ou le maintien du rôle de médiation des partis entre les publics et le gouvernement. Là aussi quelques recherches ont été menées en Europe ou aux États-Unis, alors qu'au Canada et surtout au Québec les travaux sont très rares.

Plusieurs autres champs d'étude attirent les chercheurs, mais ceux qui viennent d'être mentionnés semblent particulièrement promettre des développements nouveaux dans la recherche sur les partis et leurs systèmes. 


\section{Bibliographie}

Angell, H.M., "Le financement des partis provinciaux du Québec", in Lemieux, V. (dir.), 1982, p. 69-89.

Angell, H.M., "Duverger, Epstein and the Problem of the Mass Party : the Case of the Parti Québécois", in Revue canadienne de science politique, vol. 20, no 2, 1987 , p. 363-378.

Beaud, J.-P., «Hiérarchie partisane et sélection sociale : I'exemple du Parti québécois", in Lemieux, V. (dir.), 1982, p. 229-252.

Bélanger, R., Paul-Émile Lamarche. Le pays avant le parti (1904-1918), Sainte-Foy, Les Presses de I'Université Laval, 1984.

Bélanger, R., Wilfrid Laurier. Quand la politique devient passion, Sainte-Foy, Les Presses de I'Université Laval, 1986.

Bélanger, Y. et D. Brunelle (dir.), L'ère des libéraux. Le pouvoir fédéral de 1963 à 1984, Sillery, Presses de l'Université du Québec, 1988.

Belley, S., "Les partis politiques municipaux et les élections municipales de 1986 à Montréal et de 1989 à Québec", in Politique, no 21, hiver 1992, p. 5-35.

Benjamin, J., Comment on fabrique un premier ministre québécois, Montréal, Éditions de l'Aurore, 1975.

Bernard, A., "L'analyse politique et les partis", in Pelletier, R. (dir.), 1976, p. 19-36.

Bernier, G. et R. Boily, Le Québec en chiffres de 1850 à nos jours, Montréal, ACFAS, 1986.

Bernier, R., Le marketing gouvernemental au Québec : 1929 1985, Boucherville, Gaëtan Morin, 1988.

Bernier, R., Gérer la victoire?, Boucherville, Gaëtan Morin, 1991. 
Black, C., Duplessis (2 vol.), Montréal, Les Éditions de I'Homme, 1977.

Boily, R., "Les candidats élus et les candidats battus", in Lemieux, V. (dir.), Quatre élections provinciales au Québec : 1956-1966, Québec, Les Presses de l'Université Laval, 1969, p. 67-122.

Boily, R., "Genèse et développement des partis politiques du Québec", in Orban, E. et al., La modernisation politique du Québec, Montréal, Boréal, 1976, p. 101-144.

Boily, R., "Les partis politiques québécois - perspectives historiques", in Lemieux, V. (dir.), 1982, p. 27-68.

Boismenu, G., Le duplessisme. Politique économique et rapport de force, 1944-1960, Montréal, Presses de l'Université de Montréal, 1981.

Bourassa, G. et J. Léveillée (dir.), Le système politique de Montréal, Montréal, ACFAS, 1986.

Bourque, G. et J. Duchastel, Restons traditionnels et progressifs. Pour une nouvelle analyse du discours politique. Le cas du régime Duplessis au Québec, Montréal, Boréal, 1988.

Cardinal, M. et al., Si l'Union nationale m'était contée, Montréal, Boréal, 1978.

Comeau, P.A., Le Bloc populaire canadien, 1942-1948, Montréal, Québec/Amérique, 1982.

Comeau, R. (dir.), Jean Lesage et l'éveil d'une nation, Sillery, Presses de l'Université du Québec, 1989.

Comeau, R. et R. Dionne, Le droit de se taire. Histoire des communistes au Québec de la première guerre mondiale à la révolution tranquille, Montréal, VLB Éditeur, 1989.

Comeau, R. et L. Beaudry (dir.), André Laurendeau. Un intellectuel d'ici, Sillery, Presses de I'Université du Québec, 1990.

Dupont, A., Les relations entre l'Église et l'État sous LouisAlexandre Taschereau, 1920-1936, Montréal, Guérin, 1973.

Fraser, G., Le Parti québécois, Montréal, Libre Expression, 1984.

Gagnon, A. et A.B. Tanguay (dir.), Canadian Parties in Transition, Scarborough, Nelson, 1989. 
Gélinas, A., Les parlementaires et l'administration au Québec, Québec, Les Presses de l'Université Laval, 1969.

Hamel, J. et $\mathrm{Y}$. Thériault, *La fonction tribunitienne et la députation créditiste", in Revue canadienne de science politique, vol. 8, no 1, 1975, p. 3-21.

Jones, R., Vers une hégémonie libérale. Aperçu de la politique canadienne de Laurier King, Sainte-Foy, Les Presses de l'Université Laval, 1980.

Lafleur, G.A., PQ - PLQ, Élections 1981: Étude de stratégies électorales, Université Laval, Laboratoire d'études politiques et administratives, 1985.

Lamoureux, A., Le NPD et le Québec, 1958-1985, Montréal, Éditions du Parc, 1985.

Landry, R., "Biases in the Supply of Public Policies to Organized Interests: Some Empirical Evidence», in Coleman W.D. et G.Skogstad, (dir.), Policy Communities and Public Policy in Canada: A Structural Approach, Mississauga, Copp Clark, Pittman, 1990, p. 291-311.

Landry, R. et P. Duchesneau, «L'offre d'interventions gouvernementales aux groupes : une théorie et une application", in Revue canadienne de science politique, vol. 20, no 3, 1987, p. 525-552.

Larocque, A., Défis au Parti québécois, Montréal, Éditions du Jour, 1971.

La Terreur, M., Les tribulations des conservateurs au Québec, Québec, Les Presses de l'Université Laval, 1973.

Latouche, D., "Le contenu thématique et l'orientation idéologique des programmes électoraux de 1976», in Latouche D. et al., Le processus électoral au Québec : les élections provinciales de 1970 et 1973, Montréal, Hurtubise HMH, 1976, p. 127-150.

Legault, G. et al., Militer dans un parti provincial. Les différences entre les femmes et les hommes au PLQ et au $P Q$, Montréal, Université du Québec à Montréal, 1988. Lemieux, V., "Les partis et leurs contradictions", in Migué, J.L. (dir.), Le Québec d'aujourd'hui. Regards d'universitaires, Montréal, $\mathrm{HMH}, 1971$, p. 153-171.

Lemieux, V., "Les partis provinciaux du Québec", in Pelletier, R. (dir.), 1976, p. 53-68. 
Lemieux, V., Le patronage politique. Une étude comparative, Québec, Les Presses de I'Université Laval, 1977.

Lemieux, V. (dir.), Personnel et partis politiques au Québec, Montréal, Boréal, 1982.

Lemieux, V., Systèmes partisans et partis politiques, Sillery, Presses de l'Université du Québec, 1985.

Lemieux, V., "Le pouvoir prédominant dans les statuts des partis : le cas du NPD", in Politique, automne 1988, no 14, p. 63-82.

Lemieux, V., Les relations de pouvoir dans les lois, SainteFoy, Les Presses de I'Université Laval et I'Institut d'administration publique du Canada, 1991.

Lemieux, V., "Partis politiques et vie politique», in Daigle, G. (dir.), Le Québec en jeu, Montréal, Presses de l'Université de Montréal, 1992, p. 625-645.

Lemieux, V., Le Parti libéral du Québec. Alliances, rivalités et neutralités, Sainte-Foy, Les Presses de I'Université Laval, 1993.

Lemieux, V. et R. Hudon, Patronage et politique au Québec (1944-1972), Sillery, Boréal, 1975.

Léonard, J.F. (dir.), Georges-Émile Lapalme, Sillery, Presses de I'Université du Québec, 1988.

Maillé, C. et É. Tardy, Militer dans un parti municipal. Les différences entre les femmes et les hommes au RCM, au RP de Québec et à l'Action civique de La Salle, Montréal, Université du Québec à Montréal, 1988.

Massicotte, L., "Cohésion et dissidence à l'Assemblée nationale du Québec depuis 1867", in Revue canadienne de science politique, vol. 22, no 3, 1989, p. 505-521.

Massicotte, L., "Le financement des partis au Québec Analyse des rapports financiers de 1977 à 1989", in Seidle, F. L. (dir.), Le financement des partis et des élections de niveau provincial au Canada, vol. 3 de la collection d'études de la Commission Lortie, Toronto, Dundurn Press, 1991, p. 3-47.

Mellos, K., "Quantitative Comparison of Party Ideology", in Revue canadienne de science politique, vol. 3, no 4, 1970 , p. $540-558$. 
Monière, D., Le discours électoral. Les politiciens sont-ils fiables?, Montréal, Québec/Amérique, 1988.

Monière, D., "Analyse lexicographique du débat des chefs en français dans l'élection fédérale canadienne de 1988", in Revue canadienne de science politique, vol. 24, no 1, 1991, p. 29-50.

Murray V., Le Parti québécois. De la fondation à la prise de pouvoir, Montréal, Hurtubise HMH, 1976.

O'Neill, P. et J. Benjamin, Les mandarins du pouvoir. L'exercice du pouvoir au Québec de Jean Lesage à René Lévesque, Montréal, Québec/Amérique, 1978.

Pelletier, R., Les militants du RIN, Ottawa, Éditions de I'Université d'Ottawa, 1974.

Pelletier, R. (dir.), Partis politiques au Québec, Montréal, Hurtubise HMH, 1976.

Pelletier, R., "Les partis politiques et l'État", in Bergeron, G. et R. Pelletier (dir.), L'État du Québec en devenir, Montréal, Boréal, 1980, p. 241-261.

Pelletier, R., "Le personnel politique", in Recherches sociographiques, vol. 25, no 1, 1984, p. 83-102.

Pelletier, R., Partis politiques et société québécoise. De Duplessis à Bourassa, 1944-1970, Montréal, Québec/Amérique, 1989.

Pelletier, R., "Les structures et le fonctionnement des partis politiques canadiens", dans Bakvis, $\mathrm{H}$. (dir.), Les partis politiques au Canada : chefs, candidatset candidates et organisations, vol. 13 de la collection d'études de la Commission Lortie, Toronto, Dundurn Press, 1991, p. 299-352.

Pelletier, R. et J. Crête, "Réalignements électoraux et transformations du personnel politique", in Revue canadienne de science politique, vol. 21, no 1, 1988, p. 3-33.

Pelletier, R. et M. Tremblay, "Les femmes sont-elles candidates dans des circonscriptions perdues d'avance? De l'examen d'une croyance", in Revue canadienne de science politique, vol. 25, no 2, 1992, p. 249-267.

Pinard, M., The Rise of a Third Party, (édition augmentée), Montréal et Kingston, McGill-Queen's University Press, 1975. 
Pinard, M., «Pluralisme social et partis politiques : quelques éléments d'une théorie», in Pelletier, R. (dir.), 1976, p. 37-52.

Quesnel, L., "Un parti politique municipal : le Progrès civique de Québec*, in Pelletier, R. (dir.), 1976, p. 273-299.

Quesnel, L., "Les partis politiques locaux au Québec", in Lemieux, V. (dir.), 1982, p. 277-306.

Quesnel, L. et S. Belley, Partis politiques municipaux, Montréal, Éditions Agence d'Arc, 1991.

Renaud, F., «Les motivations dans une organisation partisane de circonscription", in Recherches sociographiques, vol. 14 , no 1,1973 , p. 59-80.

Rumilly, R., Maurice Duplessis et son temps (2 vol.), Montréal, Fides, 1973.

Stein, M.B., The Dynamics of Right-Wing Protest. A Political Analysis of Social Credit in Quebec, Toronto, University of Toronto Press, 1973.

Thomson, D.C., Jean Lesage et la révolution tranquille, SaintLaurent, Éditions du Trécarré, 1984.

Tremblay, M., «Les élues du $31^{\circ}$ Parlement du Québec et les mouvements féministes: de quelques affinités idéologiques", in Politique, automne 1989, no 16, p. 87-109.

Tremblay, M., "Quand les femmes se distinguent : féminisme et représentation politique au Québec», in Revue canadienne de science politique, vol. 25, no 1, 1992, p. 55-68.

Vigod, B.L., Quebec before Duplessis. The Political Career of Louis-Alexandre Taschereau, Montréal et Kingston, McGill-Queen's University Press, 1986. 